\title{
THE CORRELATION BETWEEN TYMPANIC MEMBRANE TEMPERATURE AND SPECIFIC REGION OF FACE TEMPERATURE
}

\author{
Dong Wook Kim ${ }^{12 \dagger}$, Ho Yeol Zhang ${ }^{13^{*} \ddagger}$, Jee-hyoung Yoo $^{4}$, Yoon Soo Park ${ }^{5}$, Hyeon Jin Song ${ }^{16}$, Kyung Hwa Yang ${ }^{16}$ \\ ${ }^{1}$ Data Center for Korean Body Temperature of National Center for Standard Reference Data (NCSRD), National \\ Health Insurance Service(NHIS) Ilsan Hospital, Goyang, Korea \\ ${ }^{2}$ Research Institute, NHIS Ilsan Hospital \\ ${ }^{3}$ Department of Neurosurgery, Spine Clinic, NHIS Ilsan Hospital \\ ${ }^{4}$ Department of Pediatrics, NHIS Ilsan Hospital \\ ${ }^{5}$ Department of Internal Medicine, NHIS Ilsan Hospital \\ ${ }^{6}$ Department of Neurosurgery, Registered Nurse, NHIS Ilsan Hospital \\ ${ }^{\dagger}$ First Author: kimdw2269@ gmail.com \\ *Corresponding Author: hyzhang@nhimc.or.kr \\ "Presenting Author: hyzhang@nhimc.or.kr
}

\begin{abstract}
Facial temperature measurements using infrared thermography (IRT) will be allowed screening travelers with high fever quickly and safely at airports or national borders if we have the formula for calculating tympanic temperature or reference standard temperature tables. This study was conducted to investigate whether the recommended position in the ISO guidelines for facial themograms measured by IRT best reflects tympanic temperature or whether other specific areas are better represented. Data were collected from NHIS Ilsan Hospital from August 2016 to December 2016. A total of 508 subjects were included in this study. The six regions of interest (ROI) of eye (medial canthus, cornea, sclera between medial canthus and cornea both side) and one ROI of inter-brow of face were defined and measured the temperature and they compared with the eardrum temperature. The results showed that the tympanic membrane temperature and medial canthus were the most similar. And a model to predict tympanic temperature through statistical bias correction can be developed.
\end{abstract}

KEYWORDS: Thermography, Medial canthus, Cornea, Sclera, Inter-brow, Bias correction

\section{INTRODUCTION}

As the population of international travel increases, the global spread of infectious diseases is accelerating. It is important to quarantine febrile travelers by measuring their body temperature quickly and safely at airports or national borders. If the relationship between the temperature of the specific region of face and the tympanic membrane temperature is measured using infrared thermography (IRT), the temperature of the traveler can be estimated. ISO guideline was published through ISO 13154:2009 with operational and implementation for screening IRT.[1] But there was no reference temperature table between facial and tympanic temperature.[2]

The purpose of this study is to evaluate the temperature of the various parts of the face and the temperature of the tympanic membrane in the inpatients of our hospital to determine which part of the face is the closest to the tympanic temperature. To improve the accuracy, we applied the statistical bias correction method.

Based on this study, we will make a relational formula of the temperature of the specific part of the face according to the room temperature, age and gender of the hospitalized patients, and reference temperature table. Based on this, we would like to develop a method of screening the body temperature of many people at airport and national borders. 


\section{MATERIAL AND METHODS}

\subsection{DESIGN AND DATA COLLECTION}

Among the patients admitted to the NHIS Ilsan Hospital, the facial thermography was taken who agreed to take our study. The tympanic membrane temperature was measured. Patients who were not able to sit or open their eyes were excluded. Facial temperature was measured by the following methods. First, the bed was curtained to block out the light and heat from the outside. Second, the patient sat on the bed, and the IR camera was placed 1.5 meters from the patient, and a thermogram was taken. Finally, left and right tympanic temperature was measured

The six regions of interest (ROI) of eye (medial canthus, cornea, sclera between medial canthus and cornea, both eyes) and one ROI of inter-brow of face were defined and measured the temperature of $4 \mathrm{X} 4$ pixels of each ROIs.

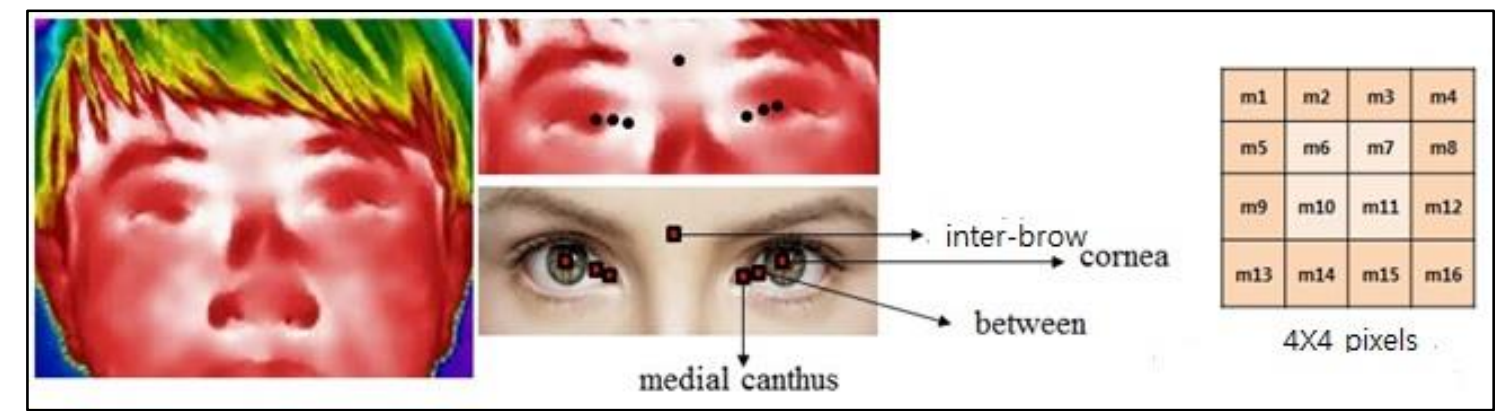

Fig. 1 Seven ROIs of temperature measurement of the eye and face. The size of each ROI was 4X4 pixels.

Patient information included in the study were gender, age, presence of lens wear, height, weight, blood pressure and diagnosis, and room temperature, humidity and time of measurement. In order to estimate the patient's temperature status from the thermal image, mean and standard deviation were calculated on the left and right medial cantus, cornea, sclera between medial canthus and cornea and inter brow by using 4 by 4 reason of interest (ROI) for each position.

\subsection{STATISTICAL ANALYSIS}

The numerical and categorical characteristics of the study population were expressed as mean \pm standard deviation and percentages, respectively. To quantitatively assess the performance of the seven spots correlation with core temperature, we calculated root mean squared error (RMSE) and quantile root mean squared error (QRMSE). In addition, we checked visual quality using by scatter plot and Taylor diagram. The scatter plots was analyzed to determine the linear relationship and the distributional identity between tympanic and thermographic temperature. The Taylor diagram was designed to compare the quantitative superiority of several thermography results. The statistical bias correction methods were used to produce internally consistent fields that have the same statistical intensity distribution as the tympanic temperature. We plan to create a model that predicts tympanic temperature by the correction method; linear regression and quantile matching method. All of graphics were created using $R$ software for Windows version 3.0.2. All of statistical measurements were performed in SAS version 9.4.

\section{RESULTS}

\subsection{BASELINE CHARACTERISITCS}

Baseline characteristics of the study population are listed in Table 1 . The mean age at the time of thermography was 48.58 years. When age were categorized by 10 years old, there were $70(13.78 \%), 51(10.04 \%), 38(7.48 \%), 22$ (4.33\%), $51(10.04 \%), 64(12.60 \%), 68(13.39 \%), 105(22.67 \%)$ and 39 (7.68\%) patients with 0 9, 10 19, 20 29, 30 39, 40 49, 50 59, 60 69, 70 79 and 80 over, respectively. Within the population, $51.97 \%$ of participants were male. Mean room temperature and humidity were $25.73{ }^{\circ} \mathrm{C}$ and $50.02 \%$, respectively. 
http://dx.doi.org/10.21611/qirt.2017.007

Table 1 Baseline characteristics of the study population.

\begin{tabular}{lr|lr}
\hline & & & \multicolumn{2}{c}{ Temperature } \\
Variable & $\mathrm{N}(\%)$ & Variable & \multicolumn{1}{c}{ Mean \pm SD } \\
\hline Sex & & Room $\left({ }^{\circ} \mathrm{C}\right)$ & $25.73 \pm 1.39$ \\
Male & $264(51.97 \%)$ & Humidity $(\%)$ & $50.02 \pm 12.71$ \\
Female & $244(48.03 \%)$ & Left & \\
Age $(\mathrm{y})$ & & Ear $\left({ }^{\circ} \mathrm{C}\right)$ & $37.21 \pm 0.46$ \\
$\leq 9$ & $70(13.78 \%)$ & Medial canthus $\left({ }^{\circ} \mathrm{C}\right)$ & $35.77 \pm 0.68$ \\
$10 \sim 19$ & $51(10.04 \%)$ & Cornea & $34.96 \pm 0.84$ \\
$20 \sim 29$ & $38(7.48 \%)$ & Between & $35.42 \pm 0.75$ \\
$30 \sim 39$ & $22(4.33 \%)$ & Right & \\
$40 \sim 49$ & $51(10.04 \%)$ & Ear $\left({ }^{\circ} \mathrm{C}\right)$ & $37.22 \pm 0.44$ \\
$50 \sim 59$ & $64(12.60 \%)$ & Medial canthus $\left({ }^{\circ} \mathrm{C}\right)$ & $35.77 \pm 0.68$ \\
$60 \sim 69$ & $68(13.39 \%)$ & Cornea $\left({ }^{\circ} \mathrm{C}\right)$ & $34.96 \pm 0.80$ \\
$70 \sim 79$ & $105(22.67 \%)$ & Between $\left({ }^{\circ} \mathrm{C}\right)$ & $35.43 \pm 0.72$ \\
$\geq 80$ & $39(7.68 \%)$ & Inter Brow $\left({ }^{\circ} \mathrm{C}\right)$ & $34.88 \pm 0.79$ \\
\hline
\end{tabular}

\subsection{QUANTITATIVE AND VISUAL QUALITY COMPARISON}

To quantitatively assess the performance of the temperature of seven ROIs, we calculated RMSE and QRMSE. The RMSE and QRMSE are defined as follows

$$
\begin{gathered}
\text { RMSE }=\sqrt{\sum \frac{\left(y_{\text {pred }}-y_{\text {true }}\right)^{2}}{n},} \\
\text { QRMSE }=\sqrt{\sum \frac{\left(\text { Rank }\left(y_{\text {pred }}\right)-\operatorname{Rank}\left(y_{\text {true }}\right)\right)^{2}}{n}},
\end{gathered}
$$

where $y_{\text {pred }}$ and $y_{\text {true }}$ denote the mean of 4 by 4 ROI and Ear temper for each person, respectively. Rank() denote the quantile-quantile of data.

Table 2 summarize the results of RMSE and QRMSE, where are obtained for with and without sweating person. As can be seen from that table, the medial canthus of the eye produce better performance result as compared to the cornea, between medial canthus and cornea and inter- brow for each side.

\begin{tabular}{|c|c|c|c|c|c|c|c|}
\hline & \multicolumn{2}{|c|}{ Total $(\mathrm{N}=508)$} & \multicolumn{2}{|c|}{ Without sweat $(\mathrm{N}=467)$} & \multicolumn{2}{|c|}{ With Sweat(N=41) } \\
\hline & & RMSE & QRMSE & RMSE & QRMSE & RMSE & QRMSE \\
\hline \multirow[t]{4}{*}{ Left } & Medial Canthus & 1.5308 & 1.4537 & 1.5417 & 1.4646 & 1.4393 & 1.3758 \\
\hline & Cornea & 2.3612 & 2.2822 & 2.3939 & 2.3152 & 2.1801 & 2.0951 \\
\hline & Between & 1.8993 & 1.8103 & 1.9175 & 1.8380 & 1.7420 & 1.6648 \\
\hline & Inter Brow & 2.4271 & 2.3603 & 2.4102 & 2.3465 & 2.6107 & 2.5290 \\
\hline \multirow[t]{4}{*}{ Right } & Medial Canthus & 1.5509 & 1.4746 & 1.5644 & 1.4882 & 1.4314 & 1.3614 \\
\hline & Cornea & 2.3575 & 2.2865 & 2.3943 & 2.3232 & 2.1303 & 2.0473 \\
\hline & Between & 1.8861 & 1.8139 & 1.9121 & 1.8406 & 1.7043 & 1.6202 \\
\hline & Inter Brow & 2.4391 & 2.3739 & 2.4231 & 2.3621 & 2.9191 & 2.5267 \\
\hline
\end{tabular}

Table 2 RMSE and QRMSE result of seven points for each tympanic temperature (Total, without and with sweat).

Figure 3 and 4 show the similarity between temperature of thermophysical image and tympanic for seven spots. The medial cantus performed well on left and right, high correlation and low RMS difference. 


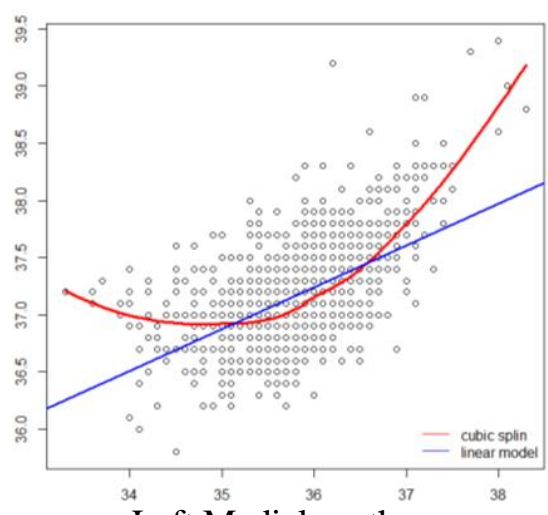

Left Medial canthus Correlation : 0.6099

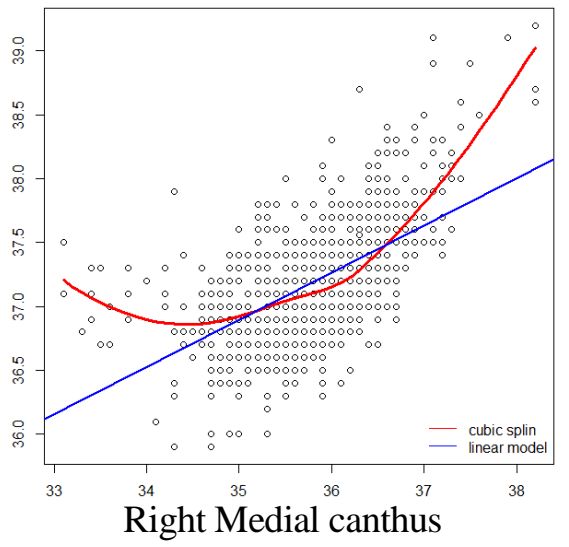

Correlation : 0.5880

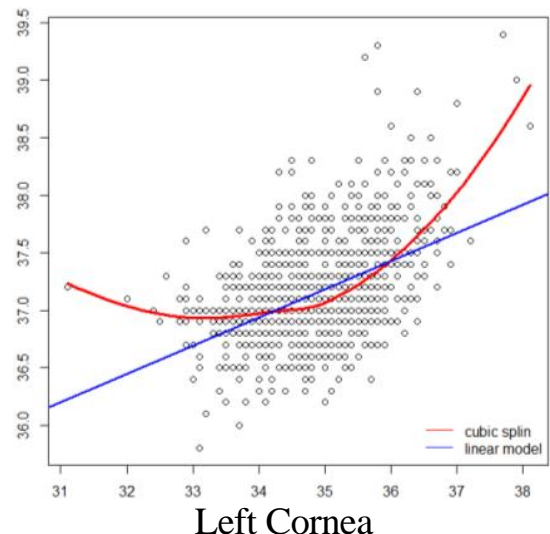

Correlation : 0.5033

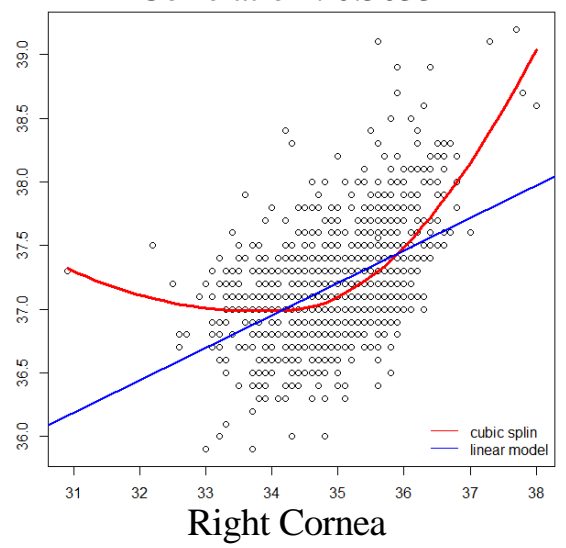

Correlation : 0.5057

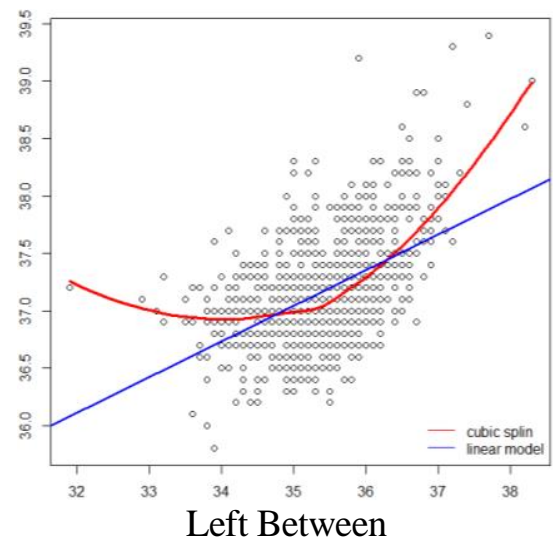

Correlation : 0.5532

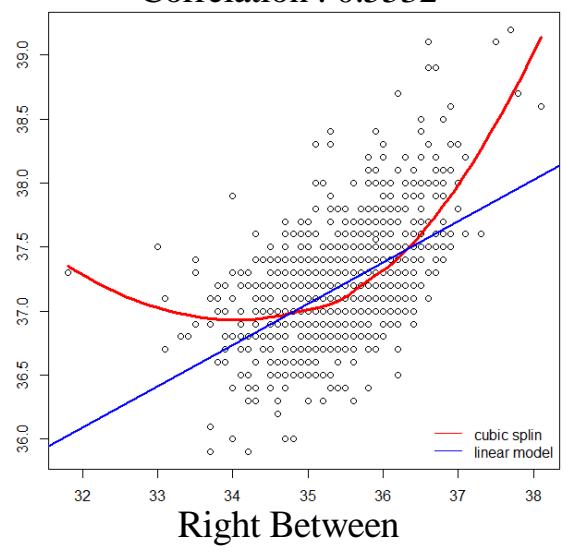

Correlation : 0.5553

Fig. 2 Scatter plot with linear regression and cubic spline for each thermography with ear temperature

(x-axis : thermography, y-axis : ear temperature)

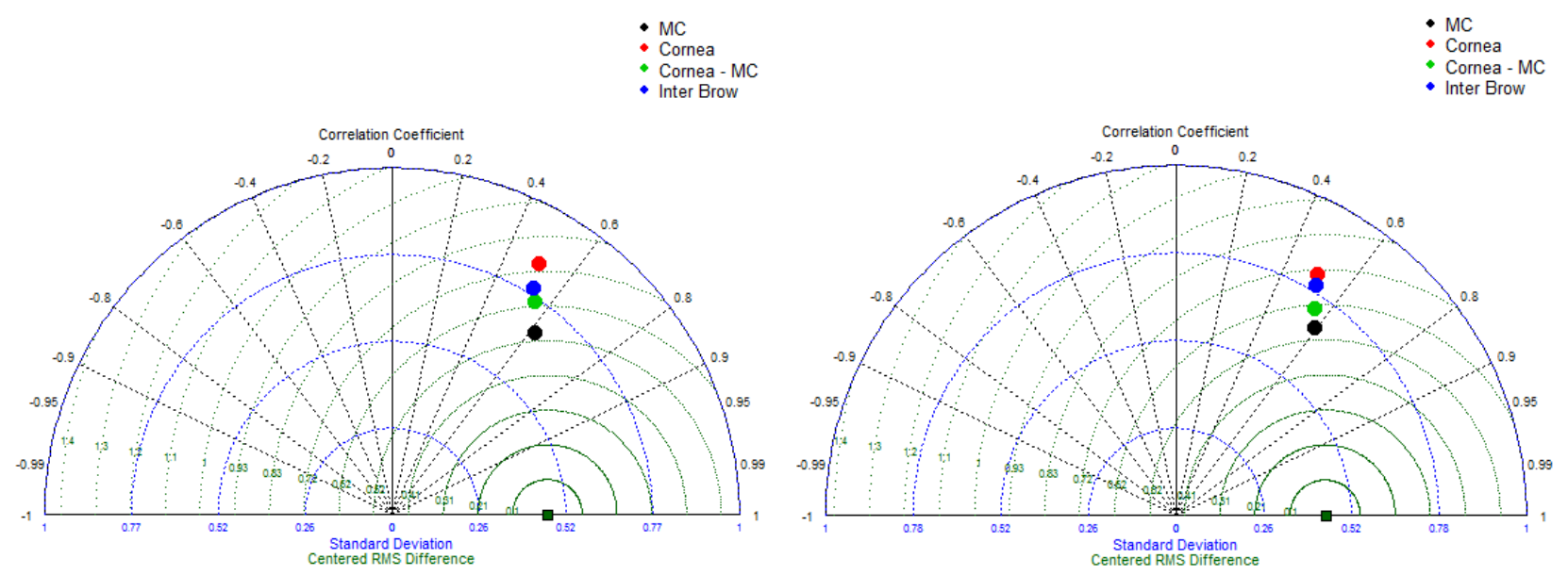

Fig. 3 The Taylor diagram compare thermography observations and ear temperature for each side.

\subsection{STATISTICAL BIAS CORRECTION}

The statistical bias correction method was used to correct the changes depending on the environment and to increase consistency with tympanic membrane temperature. The method used here is Quantile Matching.[3] RMSE and QRMSE before correction had a value of 1.0 or more both, but after then they were less than 0.5 and 0.1 respectively. Table 3 show that the RMSE and QRMSE have the best results in the medial canthus after statistical bias correction. 
Table 3 RMSE and QRMSE results of 7 points for each tympanic temperature after statistical bias correction.

\begin{tabular}{l|r|r|l|r|r}
\hline \multirow{2}{*}{ Left } & \multicolumn{2}{|c|}{ Total(N=508) } & & \multicolumn{2}{c}{ Total(N=508) } \\
\cline { 2 - 3 } \cline { 5 - 6 } Medial Canthus & RMSE & \multicolumn{1}{c|}{ QRMSE } & Right & RMSE & QRMSE \\
\hline Cornea & 0.4068 & 0.0943 & Medial Canthus & 0.3982 & 0.0923 \\
Between & 0.4590 & 0.0997 & Cornea & 0.4361 & 0.0990 \\
Inter Brow & 0.4353 & 0.0959 & Between & 0.4137 & 0.0989 \\
\hline
\end{tabular}

\section{CONCLUSIONS}

Since the face is always part of the exposed body, our study was conducted to measure the non - contact body temperature through the infrared thermography and to locate the face image similar to the tympanic temperature. According to the ISO standard, it is supposed to take the thermography the medial canthus when measuring the heat in the airport quarantine, since the temperature of the medial canthus is most similar to the temperature of the eardrum. Although it should be analyzed in detail for several measurement environments, the results showed that the face temperature position, which is the most similar to the tympanic temperature, was the medial canthus with the same result as ISO. Medial canthus makes evidence to predict the tympanic temperature by this result.

In conclusion, it is necessary to define the standard tympanic temperature according to various environments by using statistical technique and artificial intelligence technology and to search for accurate discrimination of febrile patients by matching the thermal image temperature.

\section{ACKNOWLEDGMENT}

The study was supported by the NHIS Ilsan Hospital Grant (NHIMC 2016-07-020-001). The authors alone are responsible for the content and writing of the article.

\section{REFERENCES}

[1] ISO 13154:2009. Medical reletrical equipment - deployment, implementation and operational guidelines dor identifying febrile humans using a screening thermograph

[2] C. Chandler. The use of thermography inelevated bosy temperature screenig, Pan Am J Med Thermol.2(2015) 58-62

[3] C. Piani, J.O. Haerter, E. Coppola, Statistical bias correction for daily precipitating in regional climate models over Europe, Theor Appl Climatol, 2010:pp. 187-192. 